\title{
Nutation damping of dynamically tuned gyroscopes
}

\author{
Lev Belyanin ${ }^{1, *}$, and Doan Ket $\mathrm{Vu}^{1}$ \\ ${ }^{1}$ National Research Tomsk Polytechnic University, 634050 Tomsk, Russia
}

\begin{abstract}
The paper deals with the problem of nutation damping of dynamically tuned gyroscopes (DTG). Unlike the common two-axis gyroscopes, the DTG damping torques caused by dry and viscous frictions are absent in gimbal bearings, while those due to the rotor/gaseous medium interaction are insufficient to provide the required nutation damping. A physical explanation is given to the damping torques induced in the gyroscope by the magnetoelectric torque detectors. Control coils of the latter are included in the output circuits of the electronic amplifiers. The equation is proposed for the calculation of the damping torque. The numerical estimate of the damping coefficient is given in this paper.
\end{abstract}

\section{Introduction}

An ideal configuration of a two-axis dynamically tuned gyroscope (DTG) and its ideal tuning allows considering it as a two-axis balanced gyroscope $[1,2]$.

According to the theory of a rotor two-axis gyroscope, its any forced movement is accompanied by nutation (free oscillations of a gyroscope). In common two-axis gyroscopes, nutation damping in gimbal bearings is mostly provided by the aerodynamic damping torques (interaction between the gyro case and ambient air), dry and viscous friction torques in ball bearings, and dry friction torques in the sliding current collectors and potentiometer angle transducers.

In DTG gimbal bearings the dry and viscous friction torques are practically absent, while damping torques caused by the rotor/gaseous medium interaction are low due to low viscosity and gas pressure inside the internal space of the gyroscope [3, 4]. If the torque detectors of the DTG are not involved in the operation, the damping torques turn to be insufficient to provide the appropriate nutation damping. In this case it is important to estimate the damping torques produced by the DTG torque detectors.

\section{Solution of the problem}

It is known that the damping torque component is present in a torque produced by any electric motor or torque detector. In order to obtain the equation for the numerical estimate of the damping torque in a DTG produced by the magnetoelectric torque detectors, consider

\footnotetext{
* Corresponding author: belyaninln@tpu.ru
} 
their operation at the different orientations of the rotation axis relative to the gyro case having a certain angular rate.

The formula should be derived to determine the value of the torque developed by the torque detectors, the schematic operation of which is presented in Figure 1.

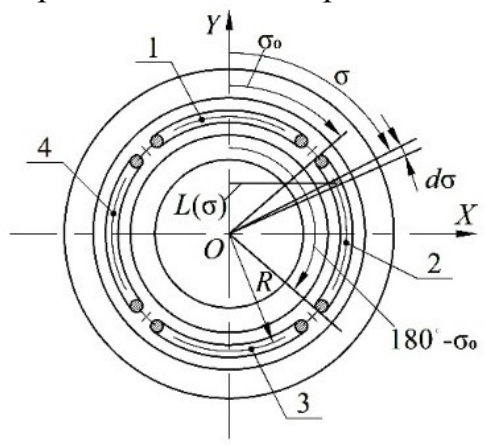

Fig. 1. Schematic operation of the torque detectors.

Figure 1 gives an example of the DTG rotor the circular groove of which allocates four coils or control windings of two magnetoelectric torque detectors. The rotor represents a constant magnet that creates a magnetic field in the circular groove, the lines of forces act along the radius. The circular groove contains the active coil parts mounted to the gyro case by their other parts. During the current flow from the coils to the conductors that form these coils and are placed normally to the rotation axis of the rotor, the axial force action is observed (Ampere's law). The coils 1 and 3 and also 2 and 4 are identical and connected to each other in series such that forces applied to the coils 1 and 3 are always directed oppositely. As a result, the created couple of forces produces the torque around the $O X$ axis. Similarly, the coils 2 and 4 create a couple of forces and the torque around the $O Y$ axis.

Let us assume that the magnetic field in the circular groove is described by the inductance $B$ the vector of which is directed along the radius, while its value is constant along the whole circle and the groove depth. Each coil has the identical number of turns equaling $w$.

The equation is then obtained for the determination of the torque produced by the torque detector and coils 2 and 4. The torque is created around the $O Y$-axis. Since these coils are completely identical and symmetrical relative to the $O Y$-axis, let us discuss only one coil. by

The force acting on the segment $d l$ of the conductor positioned at $\sigma$ angle, is determined

$$
d F=I_{t d w} \cdot B \cdot d l=I_{t d w} \cdot B \cdot R \cdot d \sigma,
$$

where $I_{t d w}$ is the current in the torque detector winding; $d l$ is the length of the segment equaling $R \cdot d \sigma$ in the angular measurement; $R$ is the radius of $d l$ section at a distance from the rotor axis.

The force $d F$ acts on $L(\sigma)$ arm and produces the torque around $O Y$-axis. It can be derived from

$$
d M_{Y}^{\prime}=d F \cdot L(\sigma)=I_{t d w} \cdot B \cdot R^{2} \cdot \sin \sigma \cdot d \sigma .
$$

The torque produced by the conductor of the coil 2 placed in the circular groove can be obtained from 


$$
M_{Y}^{\prime}=\int_{\sigma_{0}}^{180^{\circ}-\sigma_{0}} I_{t d w} \cdot B \cdot R^{2} \cdot \sin \sigma \cdot d \sigma=2 \cdot I_{t d w} \cdot B \cdot R^{2} \cdot \cos \sigma_{0} .
$$

In case the coil contains $w$ conductors and two identical coils contribute to the torque $M_{Y}$, we have

$$
M_{Y}=4 \cdot I_{t d w} \cdot w \cdot B \cdot R^{2} \cdot \cos \sigma_{0}=K_{t d} \cdot I_{t d w},
$$

where $K_{t d}=4 \cdot w \cdot B \cdot R^{2} \cdot \cos \sigma_{0}$ is the transfer factor of the torque detector.

Let us now determine the damping coefficient provided by the torque detector. For simplicity we ignore the inductance of the detector windings.

Due to the rotation around $O Y$-axis with the angular rate of $d \alpha / d t$, the winding turns of the torque detector cross the lines of forces of the magnetic field created by the constant magnet in the gap. This leads to the electromotive force in the winding turns (the law of electromagnetic induction). The value of the electromotive force de observed in the conductor can be determined from

$$
d e=\frac{d \Phi}{d t}=\frac{B \cdot d S}{d t}=B \cdot R^{2} \cdot \sin \sigma \cdot \frac{d \alpha}{d t} \cdot d \sigma
$$

where $d \Phi$ is the magnetic flux crossed by the conductor with $d l$ length; $d S$ is the area of the magnetic flux crossed by the conductor with $d l$ length during $d t$ time.

The value of the electromotive force observed over the entire length of the winding conductor is given in the following integral formulation:

$$
e(t)=\int_{\sigma_{0}}^{180^{\circ}-\sigma_{0}} B \cdot R^{2} \cdot \sin \sigma \cdot \frac{d \alpha}{d t} \cdot d \sigma=2 \cdot B \cdot R^{2} \cdot \cos \sigma \frac{d \alpha}{d t} .
$$

The electromotive force in the two opposite identical cumulative windings connected in series and containing $w$ turns each, can be obtained from

$$
d e=4 \cdot w \cdot B \cdot R^{2} \cdot \cos \sigma \frac{d \alpha}{d t}=K_{t d} \cdot \frac{d \alpha}{d t} .
$$

The electromotive force $e(t)$ acts in a closed-loop system formed by the output circuit of the amplifier, ballast resistance (if any), and ohmic resistance of the control winding. The equivalent circuit of the amplifier and winding is given in Figure 2. The amplifier output stage is assumed to be linear with the output impedance having only an active component $R_{\mathrm{ou}}$. The input is short circuited, thus $e_{\text {ou }}=0$.

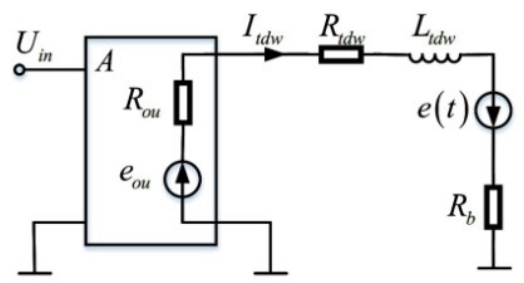

Fig. 2. Equivalent circuit of the amplifier and winding of the torque detector. 
Let define the total ohmic resistance as $R_{\Sigma}$, then the current in this circuit caused by the electromotive force $e(t)$ can be written in the following from:

$$
I_{t d w}(t)=\frac{e(t)}{R_{\Sigma}}=\frac{K_{t d}}{R_{\Sigma}} \cdot \frac{d \alpha}{d t} .
$$

The torque of the detector torque produced by this current is represented as

$$
M_{t d}(t)=K_{t d} \cdot I_{t d w}(t)=\frac{K_{t d}^{2}}{R_{\Sigma}} \cdot \frac{d \alpha}{d t} .
$$

The torque of the detector torque determined by the above written equation is just the desired damping torque. Introducing the damping coefficient provided by the torque detector we have

$$
M_{d t}=\mu \cdot \dot{\alpha},
$$

where $M_{d t}$ is the damping torque produced by the torque detector; $\mu=K_{t d}^{2} / R_{\Sigma}$ is the damping coefficient produced by the torque detector.

Let us obtain the value of the damping torque produced by the torque detector. For one of the DTG intended for the indication stabilizer systems and produced in the Russian Federation, we have $R_{t d w}=305 \mathrm{Ohm}$ and $K_{t d}=8.72 \cdot 10^{-3} \mathrm{~N} \cdot \mathrm{m} \cdot \mathrm{A}^{-1}$. Therefore, $\mu=7.6 \cdot 10^{-8} \mathrm{~N} \cdot \mathrm{m} \cdot \mathrm{s}$ at $R_{\Sigma}=1000 \mathrm{Ohm}$. In DTG intended for the production of angular rate sensors, the value of the damping coefficient provided by the torque detectors is much higher.

\section{Conclusions}

The proposed equation allowed the authors to estimate the value of nutation damping produced by the magnetoelectric torque detectors in dynamically tuned gyroscopes. The suggested numerical estimate showed that in a number of cases that damping was insufficient to suppress nutation.

\section{References}

1. D.P. Luk'yanov, V.Ya. Raspopov, Yu.V. Filatov, Principles of theory of gyroscopes (OAO Elektropribor, St-Petersburg, 2015) (in Russian)

2. G.M. Vinogradov, S.V. Krivosheev, Dynamically tuned gyroscopes (KSTU, Kazan, 2008). (in Russian)

3. A. Lawrence, Modern inertial technology: Navigation, guidance, and control (Springer-Verlag, New York, 1998)

4. Jeffrey S. Cain, Douglas A. Staley, Glenn R. Heppler, John McPhee, J. Guid. Control Dynam. 29, 965 (2006) 